\title{
Multi-stage micro rockets for robotic insects
}

\author{
Mirko Kovač, Maria Bendana, Rohit Krishnan, Jessica Burton, Micheal Smith, Robert J. Wood \\ mirko.kovac@wyss.harvard.edu,rjwood@eecs.harvard.edu \\ Micro Robotics Laboratory (http://micro.seas.harvard.edu) \\ Harvard University
}

\begin{abstract}
One of the main challenges for sustained flight of current aerial micro robots is the low energy density available from common power sources. In this paper we propose solid rocket fuel powered micro thrusters as a high energy density actuation method for aerial micro robots. In a multi stage configuration these thrusters can be used for intermittent flight which can decrease the energetic cost of locomotion. In particular we focus on the fabrication method and characterization of multistage micro thrusters with a diameter of $3 \mathrm{~mm}$ and $6.4 \mathrm{~mm}$. We demonstrate a sustained and repeatable thrust force of up to $35 \mathrm{mN}$ for a duration of up to $42 \mathrm{~s}$ and a multi-stage designs with a time delay of up to $4.7 \mathrm{~s}$ between the propulsion phases. Furthermore, we present a take-off trajectory of a $10 \mathrm{~cm}$ rocket glider with an integrated micro thruster as propulsion mechanism showing that the technologies developed can be used to successfully power micro robots in flight. Future work will focus on control and flight dynamics of micro thruster powered gliders. Wider applications of similar thrusters can include other robotic applications where low weight and high force is important such as for jumping or running robots.
\end{abstract}

\section{INTRODUCTION}

The development of flying robotic insects is one of the grand challenges in robotics research. The application of such robots includes mobile distributed sensor networks, assisted agriculture, hazardous environment exploration, and search and rescue. Several projects are underway which intend to build insect size flying robots $[1,2,3]$ but none of those systems have demonstrated sustained self-powered flight. One of the main reasons for this limitation is the high energy demand for sustained flight at small scales as well as the very low energy density of currently available power sources, such as small Lithium Polymer batteries. In order to increase the range of insect-size flying robots, we propose a hybrid flight mode as well as a novel implementation of high energy density rocket propulsion. We then integrate these micro thrusters on a new generation of robotic butterflies as illustrated in figure 1 .

Based on observations of flying animals and mathematical modeling of their flight energetics, it has been suggested that adapting a hybrid flapping-gliding flight mode, as opposed to continuous propulsion, can be a promising locomotion strategy to minimize the energetic cost of transport $[4,5]$. In fact, many animals such as birds, dragonflies, locusts, bats and butterflies employ intermittent flight, i.e. alternate propelled and gliding flight, as their aerial locomotion mode. Although recent projects have explored gliding flight for aerial micro robots $[6,7,8,9,10]$, very little research has addressed intermittent flight and its implications on the design

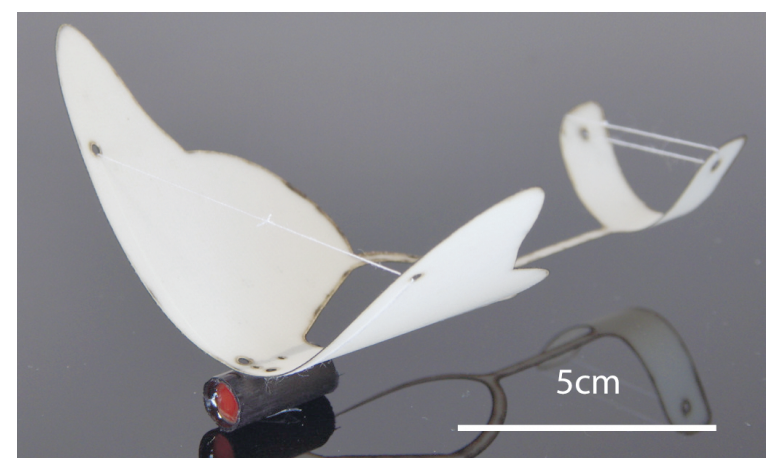

Fig. 1. Rocket glider with $10 \mathrm{~cm}$ wing span and a mass of $2 \mathrm{~g}$ with an integrated multi-stage micro thruster.

of flying micro robots. In this publication, we present our first steps towards the development of a family of multi-stage micro thrusters using solid rocket fuel that can be used to study intermittent locomotion in aerial microrobots. As micro thruster we define combustion based jet propulsion systems with a total mass of less than $10 \mathrm{~g}$. Micro thrusters are very interesting for propulsion of flying robots due to their compact and mechanically simple design, lacking moving parts which is very important for fabrication at the micro scale. Furthermore, multi-stage micro thrusters can be used to conveniently study optimal duty cycles for energy efficient intermittent flight.

The technology of micro thrusters has been developed largely with the motivation of space applications such as for nano satellites with masses in the range of $20 \mathrm{~kg}$. The goal is the generation of a very precise and repetitive propulsion that can be used to position and propel satellites $[11,12,13]$ or deploy Smart Dust sensors [14]. Other projects have focussed on optimizing combustion chamber designs of micro thrusters for use with liquid fuel [15, 16] and solid propellant [17]. Further studies have optimized wall losses and nozzle geometries for micro thrusters using numerical simulations $[18,19]$. An overview on small scale combustion-based propulsion can be found in the recent review article presented by Ju et al. [20]. Recently, Bergbreiter et al. [21] have demonstrated a jumping micro robot which uses on board combustion to initiate a jump with propellant being embedded in nano-structured silicone.

Although these projects are promising and successfully apply the technology of micro thrusters, no implementation has been presented yet which applies these technologies to flying robots. In particular, the integration of a multi-stage micro 


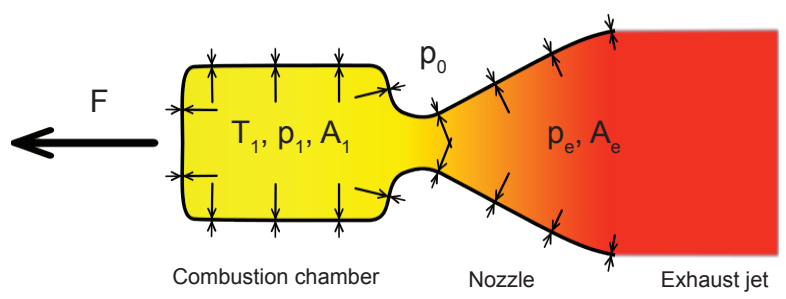

Fig. 2. Schematic representation of the combustion chamber of a rocket. $T_{c}$, $A_{c}$ and $p_{c}$ refer to the temperature, cross sectional area and pressure inside of the combustion chamber. $A_{e}$ and $p_{e}$ refer to the cross sectional area and the pressure at the end of the nozzle. $p_{0}$ is the environmental pressure.

thruster is a novelty in the field combining modulated thrust propulsion with a biologically inspired locomotion strategy.

In the coming sections, we start by presenting an overview of different options for the fuel that we considered for the micro thrusters and the rationale for the nozzle geometry that we chose. We then compare five fabrication procedures for the combustion chamber and nozzle and describe the implementation of the ignition mechanism. Furthermore, we present the micro thruster prototypes in single and multi-stage configurations and characterize their force profiles. Finally, we demonstrate successful take-off and flight of a micro thruster powered rocket glider.

\section{ROCKET PROPULSION BASICS}

Rocket propulsion engineering is a well developed and very complex field which is largely motivated by space travel and military applications. From an engineering perspective it is particularly challenging to design optimal rockets due to the interrelation of combustion physics, propellant chemistry, material science, heat transfer and phase change effects as well as fluid dynamics, including compressive flows in the supersonic nozzles. In this section we provide the basic definitions which will be referred to later when presenting the design and the results of our micro thrusters. More details on the state of the art and history of rocket design can be found in [22].

The thrust $F$ produced by a rocket depends on mass flow, exit velocity, the pressure inside the combustion chamber and in the environment as well as the cross sectional area of the nozzle. This relationship can be expressed as:

$$
F=\dot{m} v_{e}+\left(p_{e}-p_{0}\right) A_{e}
$$

where $\dot{m}$ is the changing mass due to the propellant being burned and exiting the nozzle, $v_{e}$ is the exit velocity, $p_{e}$ is the pressure of the exiting gas, $p_{0}$ is the pressure outside the rocket, and $A_{e}$ is the area ratio from the throat to the exit [22]. The first term is the momentum thrust and the second term is referred to as the pressure thrust. The throat of a rocket nozzle is defined as the smallest cross-sectional area of the nozzle. Figure 2 depicts a schematic overview of the combustion chamber and nozzle of a rocket engine. At the throat, the flow has a Mach number of one, indicating that the flow velocity equals the speed of sound which is further accelerated in the diverging section of the nozzle and typically reaches velocities of several kilometers per second.

A critical factor used to analyze the effectiveness of a rocket engine and/or a propellant is the total impulse which is defined as the thrust force integrated over the burning time:

$$
I_{s t}=\int_{0}^{t} F d t .
$$

Another important factor used to analyze the effectiveness of a rocket propulsion system is the 'specific impulse'. It is defined as the total impulse per unit weight of propellant:

$$
I_{s p}=\frac{F}{\dot{m} \cdot g}
$$

with $\dot{m}$ as the mass flow through the nozzle and $g$ as the gravitational constant. The specific impulse indicates how much propulsion was transferred from the propellant to useful thrust and it includes losses of heat through the walls, flow effects, etc. The impulse to weight ratio will be used in the coming sections to evaluate different rocket body designs. It is defined as:

$$
I_{w}=\frac{I_{s t}}{m_{c} \cdot g}
$$

with $m_{c}$ as the combustion chamber mass of the micro thruster. The impulse to weight ratio indicates which rocket construction method for the combustion chamber leads to the highest thrust produced for its weight and burn time.

\section{A. Challenges at small scales}

The most prominent effect of small scale is that the heat transfer through the walls is much more pronounced as compared to larger rockets. For larger rockets it corresponds to around 2-5\% [22] of the total energy, where for micro thrusters it can reach more than $10 \%[23,24,25]$. This is attributed to the unfavorable scaling of volume to surface area with decreasing size and has been found to be as dramatic as extinguishing the combustion due to the large heat transfer through the micro thruster walls. This effect is referred to as flame quenching and has prevented the reliable operation of silicon wafer based micro thrusters in the Smart Dust project [18]. Another effect that is pronounced at small scales is the boundary layer phenomena in the supersonic nozzle area which can lead to partial blockage of the flow and decrease in efficiency [26]. A further challenge is the fabrication and assembly of the micro thrusters. Conventional large scale systems use a variety of materials and integration methods to ensure the structural and thermal integrity of the rocket. For example, they use bolts and screws, large composite fiber layups, polymer liners to seal the inside of the combustion chamber as well as graphite inserts at the nozzle throat. Many of those fabrication methods and material choices are not possible at the scale of several millimeters or have not been developed yet. A major part of the research needed in the development of micro thrusters is therefore the material choice for the combustion chamber and nozzles as well as the choice of appropriate micro fabrication techniques. 


\section{PROPELLANT}

Various rocket propulsion technologies have been developed including liquid bi-propellants, nuclear thermal, and solid rocket propulsion. The most widely used rocket fuels for small scale systems are solid composite propellants. Its main advantage compared to liquid multicomponent fuel systems is that it is relatively easy to implement and control. The oxidizer and the fuel are both embedded in the grain of a castable solid material eliminating the need for moving parts or complex valves.

The chemical composition of the solid rocket fuel can consist of a variety of materials using different oxidizerfuel combinations and additives which act as binders and catalysts. An overview of different chemical compositions that are commonly used in existing solid fuel rocket systems can be found in [22, 27]. We choose to use Ammonium Perchlorate Composite Propellant (APCP) because of its high specific impulse, constant burning rate, good curing properties, relative ease of fabrication, and commercial availability. Higher energy fuels such as HMX are based on solid rocket fuels like APCP but increase the specific impulse by adding high-energy explosives to the mix, which leads to increased potential hazards during fabrication and operation. Its use is therefore typically limited to high performance military missiles. The specific APCP composition that we use is described in detail in [27]. Each batch contained 12\% R45-HLO resin (HTPB) as the fuel, 6\% 2-ethylhexyl acrylate (EHA) as the plasticizer, $0.5 \%$ Tepanol (HX-878) as the bonding agent, $70 \%$ ammonium perchlorate (AP, $200 \mu \mathrm{m}$ grain size) as the oxidizer, $1 \%$ ferric oxide $\left(\mathrm{Fe}_{2} \mathrm{O}_{3}\right)$ as the combustion catalyst, and $10.5 \%$ isophorone diisocyanate (IPDI) as the curing agent. The combustion temperature, and therefore the specific impulse can be increased by adding aluminum particles to the composition which act as fuel. However, this is discouraged [27] for micro thrusters because of the risk to clog the nozzle with melted aluminum particles. Additionally, a higher operation temperature would be more detrimental to the combustion chamber and would increase the risk of mechanical failure.

\section{ROCKET MOTOR DESIGN}

The design requirements for the micro thursters are dictated by the strong weight limitations and high propulsion needs of flying micro robots. The goal is to develop a combination of fabrication methods and material choices which are relatively easy to apply, allow sufficient structural and thermal stability, are light-weight and achieve a high thrust performance. In the following section we present this optimization on the design of the combustion chamber and we introduce the design choices and construction methods of the nozzle and the ignition system.

\section{A. Combustion chamber}

Combustion of the rocket fuel takes place in the combustion chamber where the chemical energy is converted to kinetic energy. The mechanical and thermal strain on the combustion chamber walls is extreme by any standards. Model rocket engines produce pressures of up to 40bar [28] at temperatures which can reach up to $2500^{\circ} \mathrm{C}$. The main requirement for the combustion chamber of the micro thruster is to be structurally resistant to this high pressure and temperature combination and have a low heat transfer coefficient in order to reduce energy losses through the combustion chamber wall. At the same time, the combustion chamber has to be as light weight as possible in order to allow for usage in flight. These design requirements lead to a trade-off between structural strength and low weight.

We optimize this trade-off by testing five different combustion chamber fabrication methods. The materials employed are carbon fiber composites and castable ceramics. As a first test, we fabricate the micro thrusters with an inner diameter of $6.4 \mathrm{~mm}$ although the same fabrication methods and materials can be used for diameters less than $2 \mathrm{~mm}$.

The first design consists of a commercially purchased protruded carbon fiber (DPP) tube with a particularly high fiber content of $63 \%$. The fiber type is Torayca T700 which is bonded using Bisphenol A Epoxy Resin. The wall thickness is $0.53 \mathrm{~mm}$ at a weight of $0.176 \mathrm{~g} / \mathrm{cm}$. These carbon fiber tubes are, to the best of our knowledge, the structurally strongest that can be purchased off the shelf at this scale and weight. However, the fibers in these tubes are oriented longitudinally at $0^{\circ}$ which is not the optimal orientation based on pressure vessel theory. In fact, it can be shown theoretically [29] that $54.74^{\circ}$ is the best fiber orientation angle with respect to the long-axis of the cylinder to sustain high internal pressures. In order to calculate this angle, let us assume a cylindrical pressure vessel which is constructed by filament winding where the fibers are laid down at a helical angle $\alpha$ as shown in figure 3. To balance the hoop and axial stresses, the tension in an infinitesimal fiber element must satisfy the following two relations which need to be solved simultaneously:

$$
\begin{gathered}
n T \sin \alpha=\frac{p r}{b}(1)(b) \\
n T \cos \alpha=\frac{p r}{2 b}(\tan \alpha)(b)
\end{gathered}
$$

with $n$ being the number of fibers, $T$ the tension in the fibers, $p$ the internal pressure and $b$ the wall thickness. After dividing the first with the second equation and rearranging we obtain:

$$
\tan ^{2} \alpha=2, \quad \alpha_{o p t}=54.74^{\circ}
$$

where $\alpha_{o p t}$ is the angle that renders the vessel twice as strong circumferentially as it is axially. More advanced models of fiber-reinforced composite pressure vessels have been developed by Parnas et. al. [30], who consider thermal loading in addition to internal pressure. The optimal angle in this case ranges between $52.1^{\circ}$ and $54.2^{\circ}$ depending on the vessel geometry and failure criteria.

The second design is a custom fabricated carbon fiber layup with an optimal winding angle of $54^{\circ}$. The fabrication method is a four step process: First, a $15.0 \mathrm{~cm}$ long sheet of $23.5 \mathrm{gsm}$ (grams per square meter) prepreg carbon fiber and C3C Cynate 


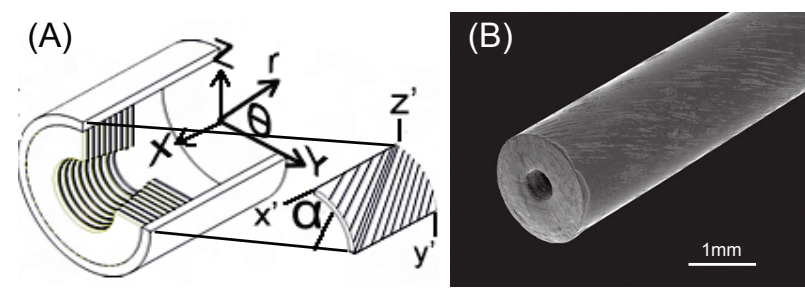

Fig. 3. The optimal fiber angle based on pressure vessel theory is $\alpha=54.7^{\circ}$. (A) Schematic for the fiber layup of the micro thrusters. (B) Fabricated micro thruster with a $54^{\circ}$ carbon fiber layup.

Ester Epoxy is cut to size, laid in a $54^{\circ}$ fiber orientation, and rolled 7 times on a metal rod which has previously been coated with Fluorinated ethylene propylene (FEP). Second, the carbon fiber sheet is placed into an FEP shrink wrap tube, secured at one end, and the air is removed. Third, the assembly is heated using a heat gun to hold the carbon fiber in place. The assembly is then placed into a high temperature oven and is heat cured at $235^{\circ} \mathrm{C}$ for 2.5 hours. Finally, the carbon fiber tube is released from the FEP shrink tube and the metal rod and is cut to the desired length. The result is a carbon tube with an inner diameter of $6.4 \mathrm{~mm}$, a wall thickness of $0.42 \mathrm{~mm}$ and a mass of $0.133 \mathrm{~g} / \mathrm{cm}$. The fiber content is $66 \%$ leading to a very resilient and light weight rocket combustion chamber.

The third design is the same as the second, but with a $45^{\circ}$ fiber angle in order to account for the effects of a possible fabrication variability on the layup angle during fabrication and to test the calculations from pressure vessel theory.

The fourth design is based on a commercial DPP tube which is dip coated on the inside in a fiber base $\mathrm{Al}_{2} \mathrm{O}_{3}$ ceramic (Cotronics Resbond 901). The properties of this ceramic are a very low thermal conductivity of only $0.22 \mathrm{~W} / \mathrm{m} \cdot \mathrm{K}$ and a high service temperature of $1430^{\circ} \mathrm{C}$. The resulting tube has a ceramic layer of $0.12 \mathrm{~mm}$ on the inside and the outside of the DPP tube. The final mass is $0.208 \mathrm{~g} / \mathrm{cm}$.

The fifth design is based on a commercial DPP tube which is spin coated on the inside wall with a single component ceramic composite based on Mica platelets and proprietary $\mathrm{Al}_{2} \mathrm{O}_{3}$ ceramic binders (Cotronics Resbond 907). Compared to Resbond 901, the 907 offers a lower service temperature of $1260^{\circ} \mathrm{C}$ and a higher thermal conductivity of $0.865 \mathrm{~W} / \mathrm{m} \cdot \mathrm{K}$. The fabricated combustion chamber has a total wall thickness of $0.53 \mathrm{~mm}$ and a mass of $0.175 \mathrm{~g} / \mathrm{cm}$.

\section{B. Ignition}

The thermodynamics of igniting composite propellants is very complex and is summarized in [22]. The combustion dynamics depends on the grain size, mixture ratios of the different components, and local heating and phase transition effects. A detailed characterization is outside the scope of this paper and the interested reader is referred to [12] which discusses this topic in detail and reviews different ignition technologies. The ignition method used for the micro thrusters in this paper is based on Joule heating with three integrated small surface mount resistors (figure 4). The complete igniter

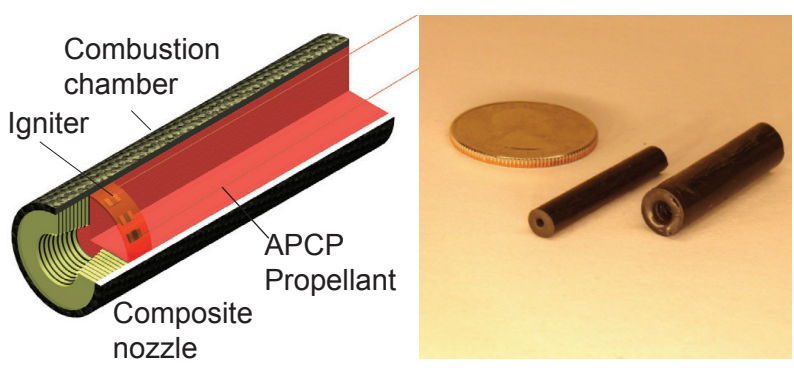

Fig. 4. The fabricated micro thruster has build in ignition and a high strength composite nozzle. The fabrication method allows fabricating micro thrusters with sizes of less than $1.5 \mathrm{~mm}$.

has a mass of only $0.12 \mathrm{~g}$. Reliable ignition is achieved and requires heating power of $2 \mathrm{~W}$ for a duration of $1 \mathrm{~s}$. A $0.3 \mathrm{~g}$ $10 \mathrm{mAh}$ LiPo battery can theoretically sustain $2 \mathrm{~W}$ for $7.2 \mathrm{~s}$ and could be used to ignite the micro thrusters. More energy efficient igniter solutions are possible by using electrically activated pyrotechnic initiators. Common compositions of such initiators consist of zirconium-potassium perchlorate or boronpotassium nitrate.

\section{Nozzle}

The purpose of the expanding section of the nozzle is to further accelerate the flow after the throat area and create positive pressure which can be used to increase thrust.

The main requirement for the nozzle wall is structural and thermal stability during operation and a smooth surface allowing for sustained flow attachment. The nozzle wall should therefore be smooth and have a smooth transition from the throat to the nozzle in order to avoid abrupt pressure changes which could lead to shock waves which can choke the flow through the nozzle and decrease thrust. Traditionally, rocket nozzles are made from high temperature steel or fiber composite materials, with graphite insertions at high abrasion areas such as the throat [22]. A nozzle opening angle of $30^{\circ}$ is an empirical design standard and has been determined to be optimal for micro thrusters [19] using numerical simulations.

The nozzle for the micro thrusters presented in this paper have been designed to be (i) light weight, (ii) easy to fabricate at small scales, (iii) structurally resistant at high temperatures and (iv) easy to integrate with the combustion chamber. The chosen design uses layered glass fiber as the structural material with a high thermal resistance, high temperature epoxy as the bonding agent to integrate the glass fiber mesh and graphite grains to increase the thermal resistance of the composite. A cut CAD design of a nozzle is depicted in figure 5.A. The fabrication process begins by machining twelve rings of $0.128 \mathrm{~mm}$ wear resistant FR4 sheets using a $\mathrm{CO}_{2}$ laser (Versa Laser 2.3). The rings are assembled using a precision pin alignment method [31]. The layers are laminated using high temperature epoxy with $20 \%$ graphite powder for two hours at $120^{\circ} \mathrm{C}$ and four hours at $235^{\circ} \mathrm{C}$ under a weight of $50 \mathrm{kN} / \mathrm{m}^{2}$. Subsequently, the nozzle is released from its pin alignment structure and is ready to be integrated with the combustion 


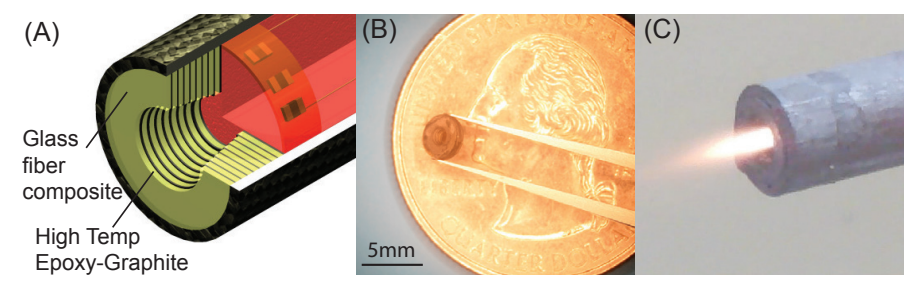

Fig. 5. The nozzle consist of a combination of glass fiber laminate with embedded high temperature epoxy and graphite to for high strength and structural integrity at high temperatures. A: CAD design, B: Fabricated nozzles with a U.S. quarter coin for size comparison, C: Micro thruster in operation.

chamber. The final fabricated nozzles can be seen in figure 5.B on a U.S. quarter coin for size reference. A Scanning Electron Microscope (SEM) image of the fabricated nozzle is depicted in figure 5.C. Once the nozzle is fabricated, it is embedded into the combustion chamber using the same high temperature epoxy graphite mix adhesive. The final rocket motor in operation is shown in figure 5.D.

\section{Multi-stage design}

The multi-stage design enables thrust modulation by reducing the thrust force temporarily over a fraction of a second to several seconds. We considered several methods where the burning rate of the propellant is modulated using a combustion cooler, such as sodium bicarbonate, which reduced the thrust output but at the cost of a less stable combustion. Other methods include hybrid propellant designs with a low performance propellant inserted between the stages. In particular, we tested potassium nitrate mixed with sulfur and charcoal compositions which are also used as the delay charge in model rockets. We also tested potassium nitrate sucrose composite propellant which is a common hobbyist rocket fuel and provides a lower specific impulse than APCP. The main challenge in such a design was to ensure flame transition between the different propellants where each has a different flame and melting temperature and burning rate. In addition, the drawback is that a fraction of the propellant is intentionally designed to give little thrust which adds weight to the micro thruster without adding impulse to it.

After evaluating the methods described above, we decided to opt for a novel multistage design where we modify the geometry of the combustion chamber by introducing combustable delay charges which allow for temporal thrust modulation. The delay charge consists of layered cellulose fiber rings which are glued together using acetone-based spray adhesive. A three stage micro thruster design is illustrated in figure 6 . The inner ring of the delay charge is $2 \mathrm{~mm}$ in diameter and filled with APCP which ensures that the combustion continues between the two stages. The delay charge itself is highly combustable and reduces the thrust force for a limited time due to the temporal change in combustion chamber geometry. As soon as the second stage ignites, it burns away and allows the second stage to use the entire combustion chamber. The main advantage of this method for the delay charge design is that it is very simple to integrate and that it allows for a large

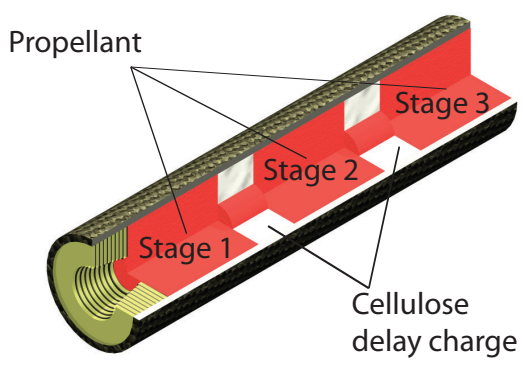

Fig. 6. Micro thruster in a multi-stage configuration.

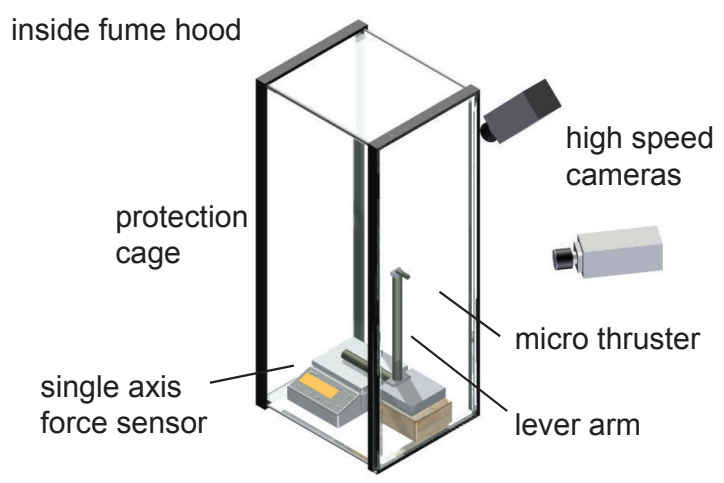

Fig. 7. The experimental setup allows measuring the thrust force of the micro thrusters in a controlled and enclosed environment.

range of thrust modulation by changing the inner ring diameter and thickness of the delay charge. Further, it does not require a flame transition between different propellant compositions which reduces the risk of flame extinction at the interface of the delay charge and the main propellant.

\section{Results}

The experimental setup (figure 7) is enclosed in a fume hood in order to contain the exhaust gases of the micro thruster. The micro thruster is clamped on a carbon fiber lever arm which rotates using a low friction ball bearing around the axis. The lever arm is connected to a computer controlled single axis force sensor which has a resolution of $0.1 \mathrm{mN}$ and a measurement bandwidth of $10 \mathrm{~Hz}$. The lever arm and the force sensor are encased in a transparent protective cage to reduce the measurement noise due to the air flow caused by the ventilation system of the fume hood. The impulse is calculated by integrating the thrust force over time as described in section II. In this section we start by comparing the performance of the different combustion chamber fabrication methods and materials. Further, we characterize the scaling behavior of the micro thrusters by fabricating and testing different lengths and diameters. Finally, we characterize multi-stage configurations and demonstrate successful take-off and flight of a $10 \mathrm{~cm}$ rocket glider with micro thruster based propulsion.

\section{A. Combustion chamber materials}

In order to decide which combustion chamber fabrication method and material is best for the application of intermittent 


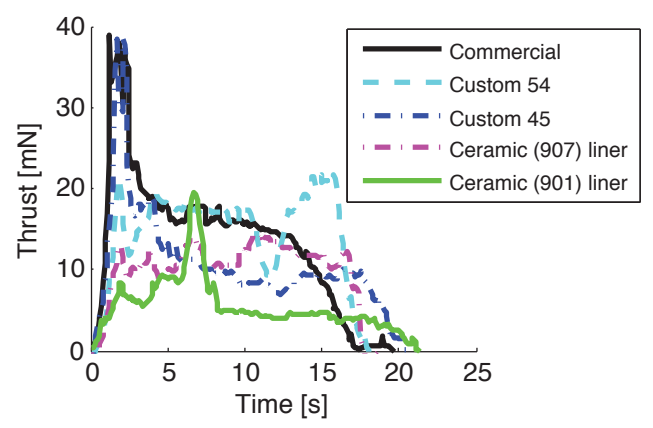

Fig. 8. Force profile for the combustion chamber fabricated with different materials an fiber layup angles. The force profiles are more erratic for the ceramic liners indicating irregular combustion.

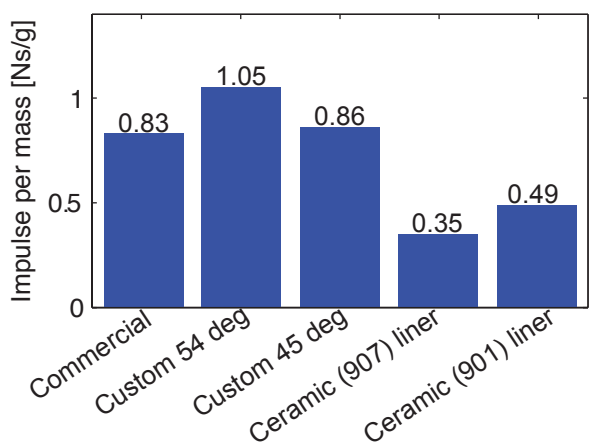

Fig. 9. The design with a $54.7^{\circ}$ fiber orientation exhibits the best impulse to mass ratio, $21 \%$ higher than the commercially purchased carbon fiber tubes.

flight for small robots, we measure the impulse per mass for every motor using the same amount of propellant. Each combustion chamber is filled with $1.4 \mathrm{~g}$ of APCP. The force profiles for the different designs are shown in figure 8 and the impulse to mass ratio in figure 9 . It can be seen that the design with a $54.7^{\circ}$ fiber orientation exhibits the best impulse to mass ratio, $21 \%$ higher than the commercially purchased carbon fiber tubes. The ceramic coatings do not improve the impulse to mass ratio. Furthermore, the force profiles are more erratic, indicating irregular combustion which we suspect to be caused by flaking of the ceramic liner during combustion.

\section{B. Scaling}

In order to characterize the scaling behavior and repeatability of the micro thrusters we fabricate samples with lengths between $15 \mathrm{~mm}$ and $60 \mathrm{~mm}$ for the micro thruster with $6.4 \mathrm{~mm}$ (figure figure 10.A) and with $3 \mathrm{~mm}$ diameter (figure 10.B). It can be seen that all lengths have a similar maximal thrust plateau of around $20 \mathrm{mN}$ for the larger thrusters and $8 \mathrm{mN}$ for the smaller design with an initial force peak at the start of the burn. This initial force overshoot is a known phenomena and is attributed to the surface area of the flame front. It peaks as it becomes a cone coming out of the nozzle into the body and then flattens out as it hits the body casing and burns at a constant rate. The maximal burn duration for the
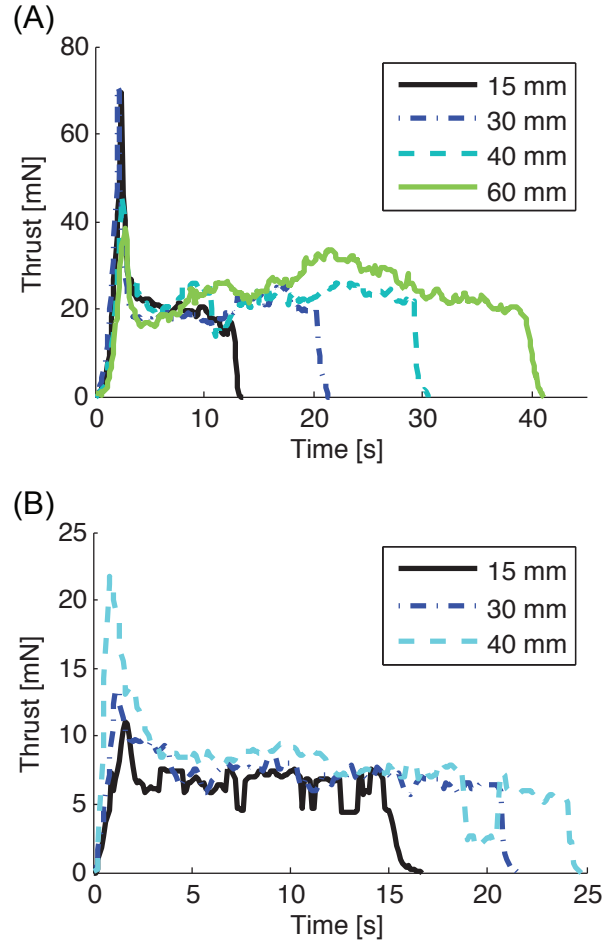

Fig. 10. Force profiles for different lengths for the micro thrusters with $6.4 \mathrm{~mm}$ diameter $(\mathrm{A})$ and $3 \mathrm{~mm}$ diameter $(\mathrm{B})$. The maximal thrust duration of a $60 \mathrm{~mm}$ micro thruster is $42 \mathrm{~s}$.

$60 \mathrm{~mm}$ thruster is $42 \mathrm{~s}$ with a slightly convex force profile. The convex shape could be caused by a build up of pressure and temperature during the first 20 s which increases the burning rate of the propellant. This then decreases as the volume becomes larger with a higher percentage of heat loss through the walls which slightly cools the combustion, producing less force.

\section{Multi-stage micro thruster}

We characterize the thrust modulation by varying the delay charge thickness between $1.29 \mathrm{~mm}$ and $3.67 \mathrm{~mm}$ using the micro thrusters with $6.4 \mathrm{~mm}$ diameter. For statistical relevance we fabricate and test three samples at each delay charge thickness and plot the average force profile for each case and the standard deviation in figure 11.A. The average time where the thrust is reduced below $10 \mathrm{mN}$ is plotted in $11 . \mathrm{B}$ as a function of the delay charge thickness. As a proof of concept for multistage designs, we fabricate a three stage micro thruster and measure the force profiles (figure 11.C). These results demonstrate that the thrust can be successfully modulated with a time delay of up to $4.7 \mathrm{sec}$ and a complete thrust recovery at the following thrust phase. Further, we demonstrate that three stage micro thrusters are feasible using the presented fabrication and integration methods.

\section{Integration on a microglider}

As a first step towards the integration of these micro thrusters on a micro glider testbed we fabricate and attach a 

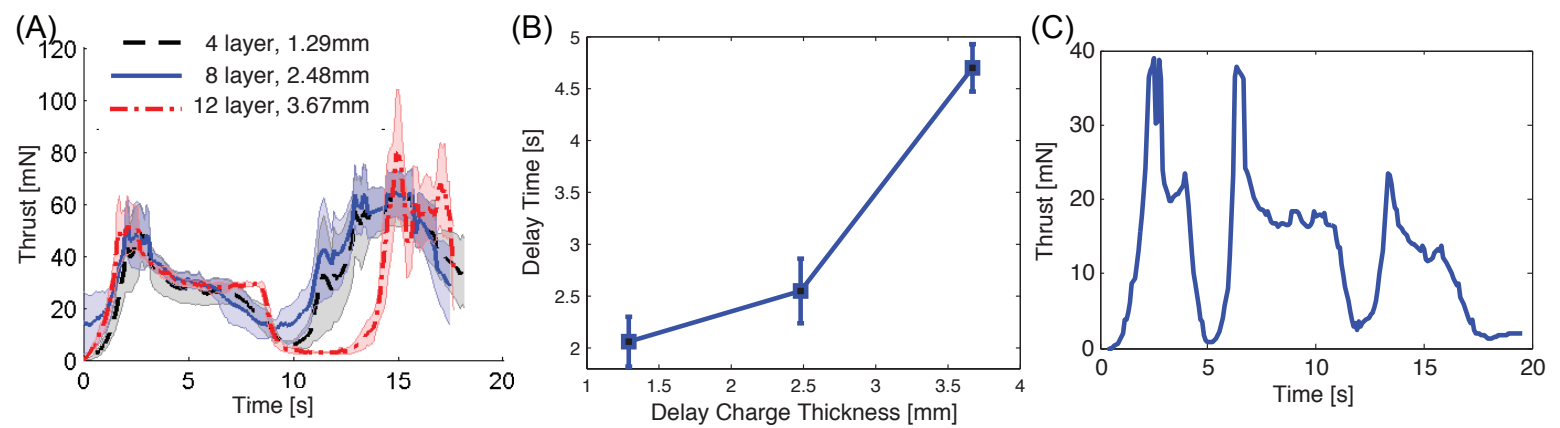

Fig. 11. The multi-stage configuration allows thrust modulation with a delay time of up to $4.7 \mathrm{~s}$ between the propulsion phases. A: Thrust force results for different delay charge thicknesses. Each thickness has been repeated three times, the filled line represents the mean value the shaded area the standard deviation, B: Delay charge thickness vs. measured time delay, C: Thrust profile of a three stage micro thruster.

single stage micro thruster to a micro glider with a wingspan of $10 \mathrm{~cm}$ (figure 1). The wing loading of this glider is $5.5 \mathrm{~N} / \mathrm{m}^{2}$ which is in the same order of magnitude as large butterflies or bats. As wing material we use glass fiber reinforced epoxy FR4 laminate sheets with a thickness of $250 u \mathrm{~m}$. A sample flight trajectory is illustrated in figure 12 where we test the robustness of the glider material to the very high thermal load on the micro thruster body as well as the force acting on the glider. The glider performs a successful and stable take-off from a starting ramp, although it is not yet fully balanced which leads to a pitch instability after around $500 \mathrm{~ms}$ flight time. These results indicate that the chosen glider materials and integration methods are suitable for micro thruster powered gliders and that the developed micro thrusters have largely sufficient thrust to propel flying micro robots with a mass of several grams. Future work will focus on multi stage designs and control of the rocket gliders using piezo or Shape Memory Alloy based steering actuators such as in $[8,10]$.

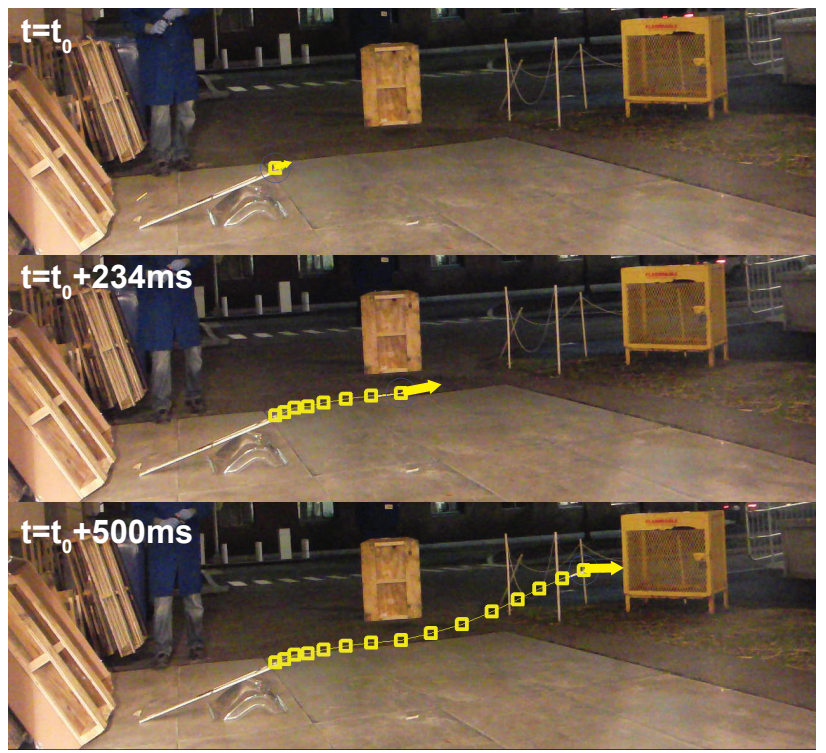

Fig. 12. Successful micro thruster powered take-off trajectory of a $10 \mathrm{~cm}$ rocket glider with a mass of $2 \mathrm{~g}$.

\section{CONCLUSION AND FUTURE WORK}

In this paper we presented the development and integration of novel multi-stage micro thrusters which can be used for the propulsion of the next generation of energy efficient micro robots. We demonstrate a sustained and repeatable thrust force of up to $35 \mathrm{mN}$ for a duration of up to $42 \mathrm{~s}$ and multi-stage designs with a time delay of up to $4.7 \mathrm{~s}$ between the propulsion phases. The average specific impulse for the developed micro thrusters is 27.2s. Based on the characterization and the successful integration and take-off trajectory of these micro thrusters on a glider testbed, we conclude that solid rocket propulsion is a promising actuation method for robotic insects. It can as well be used for other systems which require high force and low weight such as for jumping or running robots. Future work will focus on the mathematical modeling and experimental characterization of the flight phases of rocket gliders to study optimal duty cycles for intermittent flight.

\section{ACKNOWLEDGMENTS}

The authors would like to thank Prof. J. C. Oxley and Dr. Patrick Bowden from Rhode Island University for the helpful discussions on solid rocket chemistry as well as Nelly Briquet for the assistance with the thruster experiments. Furthermore, we thank Steve Sansone from the particle accelerator facility to conduct our flight tests there. We would also like to thank Dr. James Weaver for taking the SEM images of the nozzles. This work is partially funded by the Wyss Institute for Biologically Inspired Engineering (award number ONR N00014-10-1-0684).

\section{REFERENCES}

[1] R. J. Wood, "The first takeoff of a biologically inspired at-scale robotic insect," IEEE Transactions on Robotics, vol. 24, no. 2, pp. 341-347, 2008.

[2] V. Arabagi and M. Sitti, "Simulation and analysis of a passive pitch reversal flapping wing mechanism for an aerial robotic platform," in IEEE/RSJ International Conference on Intelligent Robots and Systems, 2008, pp. 1260-1265.

[3] H. Tanaka, K. Matsumoto, and I. Shimoyama, "Design and performance of micromolded plastic butterfly wings 
on butterfly ornithopter," in IEEE/RSJ International Conference on Intelligent Robots and Systems, 2008, pp. 3095-3100.

[4] J. Rayner, P. W. Viscardi, S. Ward, and J. R. Speakman, "Aerodynamics and energetics of intermittent flight in birds," American Zoologist, vol. 41, no. 2, pp. 188-204, 2001.

[5] J. J. Videler, D. Weihs, and S. Daan, "Intermittent gliding in the hunting flight of the kestrel Falco Tinnunculus," Journal of Experimental Biologyiology, vol. 102, pp. 1$12,1983$.

[6] K. Peterson, P. Birkmeyer, R. Dudley, and R. S. Fearing, "A wing-assisted running robot and implications for avian flight evolution," Bioinspiration \& Biomimetics, vol. 6, 2011.

[7] M. Kovac, W. Hraiz, O. Fauria, J.-C. Zufferey, and D. Floreano, "The EPFL jumpglider: A hybrid jumping and gliding robot," in IEEE International Conference on Robotics and Biomimetics, 2011, pp. 1503-1508.

[8] R. J. Wood, S. Avadhanula, E. Steltz, M. Seeman, J. Entwistle, A. Bachrach, G. L. Barrows, S. Sanders, and R. S. Fearing, "Design, fabrication and initial results of a $2 \mathrm{~g}$ autonomous glider," in IEEE Industrial Electronics Society Meeting, 2005.

[9] M. Woodward and M. Sitti, "Design of a miniature integrated multi-modal jumping and gliding robot," in IEEE/RSJ International Conference on Robotics and Automation, 2011, pp. 556 - 561.

[10] M. Kovac, A. Guignard, J.-D. Nicoud, J.-C. Zufferey, and D. Floreano, "A $1.5 \mathrm{~g}$ sma-actuated microglider looking for the light," in IEEE International Conference on Robotics and Automation, 2007, pp. 367-372.

[11] B. Larangot, V. Conedera, P. Dubreuil, T. Do Conto, and C. Rossi, "Solid propellant microthruster: An alternative propulsion device for nanosatellite," in Aerospace Energetic Equipment Conference, 2002.

[12] D. A. De Koninck, U. Bley, V. Gass, D. Briand, and N. F. De Rooij, "Pyromems igniter based on a temperature gradient: Concept, fabrication and characterization," in Proceedings of the 9th International Workshop on Micro and Nanotechnology for Power Generation and Energy Conversion Applications, vol. 1, 2009, pp. 108-111.

[13] K. L. Zhang, S. K. Chou, S. S. Ang, and X. S. Tang, "A mems-based solid propellant microthruster with au/ti igniter," Sensors and Actuators A: Physical, vol. 122, no. 1, pp. 113-123, 2005.

[14] D. Teasdale and K. S. J. Pister, "Solid propellant microrockets," Master's thesis, University of California at Berkeley, 2000.

[15] M. Wu, Y. Wang, R. Yetter, and V. Yang, "Liquid monopropellant combustion in mesoscale vortex chamber," Journal of propulsion and power, vol. 25, no. 3, 2009.

[16] L. Sitzki, K. Borer, E. Schuster, P. D. Ronney, and S. Wussow, "Combustion in microscale heat-recirculating burners," in The Third Asia-Pacific Conference on Combustion, vol. 6, 2001, pp. 11-14.
[17] E. A. Parra, K. S. J. Pister, and C. Fernandez-Pello, "A practical solid-propellant micro-thruster," in ASME International Mechanical Engineering Congress and Exposition, 2006.

[18] E. Rudnyi, T. Bechtold, J. Korvink, and C. Rossi, "Solid propellant microthruster: Theory of operation and modelling strategy," Proc. Nanotech'02, AIAA paper 2002, vol. 5755, 2002.

[19] I. Kim, J. Lee, M. Choi, and S. Kwon, "Optimum nozzle angle of a micro solid-propellant thruster," Nanoscale and Microscale Thermophysical Engineering, vol. 15, no. 3, pp. 165-178, 2011.

[20] Y. Ju and K. Maruta, "Microscale combustion: Technology development and fundamental research," Progress in Energy and Combustion Science, vol. 1, no. 1, pp. 1-47, 2011.

[21] W. A. Churaman, A. P. Gerratt, and S. Bergbreiter, "First leaps toward jumping microrobots," in IEEE/RSJ International Conference on Intelligent Robots and Systems, 2011, pp. 1680-1686.

[22] G. P. Sutton, Rocket propulsion elements-An introduction to the engineering of rockets. New York, WileyInterscience, 1992, vol. 1.

[23] V. Shirsat and A. K. Gupta, "A review of progress in heat recirculating meso-scale combustors," Applied Energy, vol. 88, no. 12, pp. 4294-4309, 2011.

[24] K. L. Zhang and S. S. Chou, S. K.and Ang, "Performance prediction of a novel solid-propellant microthruster," Journal of propulsion and power, vol. 22, no. 1, pp. 5664, 2006.

[25] H. Mirels, "Effect of wall on impulse of solid propellant driven millimeter-scale thrusters," AIAA journal, vol. 37, no. 12, pp. 1617-1624, 1999.

[26] R. L. Bayt and K. S. Breuer, "Viscous effects in supersonic mems-fabricated micronozzles," in Proceedings of the 3rd ASME Microfluids Symposium, 1998, pp. 117123.

[27] G. W. Purrington, Plastic Resin Bonded High Energy Rocket Fuel Systems. Basic Ingredient Study and Small Motor Production. Vol III. Firefox Enterprises, 2001.

[28] L. H. Hertz, The complete book of model aircraft, spacecraft and rockets. Crown Publishers, 1967.

[29] P. M. Wild and G. W. Vickers, "Analysis of filamentwound cylindrical shells under combined centrifugal, pressure and axial loading," Composites Part A: Applied Science and Manufacturing, vol. 28, no. 1, pp. 47-55, 1997.

[30] L. Parnas and N. Katirci, "Design of fiber-reinforced composite pressure vessels under various loading conditions," Composite Structures, vol. 58, no. 1, pp. 83-95, 2002.

[31] J. P. Whitney, P. S. Sreetharan, K. Y. Ma, and R. J. Wood, "Pop-up book mems," Journal of Micromechanics and Microengineering, vol. 21, no. 11, pp. 115021-115027, 2011. 